\title{
Application of the Type, Entry Site, and Malperfusion Classification in Treatment of Aortic Dissection
}

\author{
Xuening Wang, MD, PhD,${ }^{1 *}$ Chunyan Wang, MD, PhD,${ }^{2 *}$ Xinmeng Cheng, MD, ${ }^{3}$ Yuyuan Hu, MD, \\ Lingbo Yang, $\mathrm{MD},{ }^{1}$ William Wang, $\mathrm{MD}, \mathrm{PhD}^{4}$
}

\begin{abstract}
${ }^{1}$ Department of Cardiovascular Surgery, Shanxi Academy of Medical Sciences \& Shanxi Bethune Hospital, Third Hospital of Shanxi Medical University, Tongji Shanxi Hospital, Taiyuan, Shanxi, China; ${ }^{2}$ Department of Molecular Biology, Shanxi Cancer Hospital and Institute, Taiyuan, Shanxi, China; ${ }^{3}$ Department of Cardiovascular Surgery, Third Hospital of Shanxi Medical University, Tongji Shanxi Hospital, Taiyuan, Shanxi, China; ${ }^{4}$ Department of Cardiac Surgery, Scripps Memorial Hospital, La Jolla, CA, USA
\end{abstract}

\section{ABSTRACT}

Background: Our goal is to investigate a new practical dissection classification system, including type of dissection, location of the tear of the primary entry, and malperfusion.

Methods: The outcome of 151 patients with aortic dissection between January 2019 and May 2020 retrospectively were analyzed. All cases were classified with the Stanford dissection classification (A and B) by adding type non-A non-B. They were then further classified by the new classification system, including location of the primary Entry (E) and Malperfusion $(\mathrm{M})$. All cases were followed up for six months.

Results: The distribution of 151 patients was $53.0 \%, 27.8 \%$, and $19.2 \%$, respectively, in type A, B, and non-A non-B. The in-hospital mortality rate was $8.8 \%, 2.4 \%$, and $3.4 \%$ in type A, $\mathrm{B}$, and non-A non-B $(P<0.05)$ and postoperative neurological complications occurred in $33.8 \%, 7.1 \%$, and $13.8 \%$ in type A, $\mathrm{B}$, and non-A non-B $(P<0.05)$. Total arch replacement was performed in $53.8 \%, 4.8 \%$, and $13.8 \%$ in type $\mathrm{A}, \mathrm{B}$, and non-A non-B. The in-hospital mortality rate was $12.0 \%, 10.4 \%$, and $8.5 \%$ in type E1, E2 and E3, while it was $20.0 \%, 10.4 \%$, and $8.5 \%$ in type $M 1, M 2$ and M3 $(P<0.05)$.

Conclusions: The new practical dissection classification system is useful as a supplement to the Stanford dissection classification by regarding the extent of the disease process, aiding in decision-making about the operative indication and plan, and helping in anticipating prognosis.

\section{INTRODUCTION}

Aortic dissection is the most common catastrophic and complex disease in aortic diseases. The two most commonly used classifications for aortic dissection are the Stanford and Debakey systems, introduced in the 1950s and 1960s

Received fuly 4, 2021; accepted August 9, 2021.

${ }^{*}$ Contributed equally as co-first authors.

Correspondence: Xuening Wang, Department of Cardiovascular Surgery, Shanxi Academy of Medical Sciences \& Shanxi Bethune Hospital, Taiyuan, Shanxi, China, Telephone (+86)150351 18416 (e-mail: wangxuening_2004@126.com).
[Daily 1970; Colli 2018]. By definition, the Stanford classification system divides aortic dissection into type A and type B. Futhermore, the Stanford type A dissection involves the ascending aorta, and type B is limited to the descending aorta, below the left subclavian artery. The DeBakey classification is similarly focused on ascending and descending involvement in the dissection process. However, both of the classifications are irrespective of the location of the primary entry tear and end-organ malperfusion, which play an important role in aortic dissection diagnosis and treatment procedure. With improvements in imaging techniques, such as computed tomographic angiography (CTA) and magnetic resonance, aortic dissection is currently diagnosed more accurately. And, with great progress of techniques, such as surgery, intervention, and hybrid procedures, more and more attention has been paid to the primary entry [Colli 2018; Shi 2016; Khoynezhad 2010; Weiss 2012] and end-organ malperfusion [Geirsson 2007; Kreibich 2019; Di Eusanio 2013].

Based on the Stanford classification, a novel classification system for aortic dissection, named TEM system, which includes type, entry location and malperfusion (TEM) status was introduced by Sievers et al. [Sievers 2020]. The TEM classification provides additional information on dissection anatomy and end-organ malperfusion, which could help to define the therapeutic option and predict outcomes. The objective of this study is to apply and analyze the outcome of the new dissection classification.

\section{MATERIALS AND METHODS}

Patients: A total of 151 cases with aortic dissection were treated in our hospital, during the period of January 2019 through May 2020. All patients immediately received CTA scans after onset of dissection and were classified by dissection anatomy and clinical symptoms. All cases were followed up for six months. The institutional review committee approved this retrospective study, and the need for informed consent was waived.

Application of the type, entry and malperfusion classification system - Type: The new practical classification system was introduced by Sievers in 2020, which is based on the aortic dissection's extension, the entry location and 
end-organ malperfusion [Sievers 2020]. Based on the Stanford classification, aortic dissection was classified into three main categories, according to the dissection's extension, Type A, Type B, and Type non-A non-B [Sievers 2020]. Dissection involving the ascending aorta with or without extension into the aortic arch and descending aorta was classified into Type A. Dissection involving the descending aorta but not the aortic arch or ascending aorta was considered Type B. And dissection involving the aortic arch and the descending aorta but not the ascending aorta was named Type non-A non-B.

Application of the type, entry and malperfusion classification system - Entry: The entry location was found by CTA scans and reconfirmed during operation or interventional radiography. According to the new classification, the location of primary entry is characterized as follows: E0 (no entry), E1 (entry in the ascending aorta between the aortic valve and the proximal edge of the brachiocephalic trunk offspring), E2 (entry in the aortic arch between the proximal edge of the brachiocephalic trunk offspring and the distal edge of the left subclavian artery offspring), and E3 (entry in the descending aorta, below the distal edge of the left subclavian artery offspring).

Malperfusion: The end-organ malperfusion, according to imaging findings and clinical signs, is characterized as follows: M0 (no radiological or clinical signs of malperfusion); M1 (dissection of at least 1 main coronary artery with (M1+) or without (M1-) indicators of cardiac ischaemia, such as electrocardiographic and blood test abnormalities typical for myocardial malperfusion); M2 (dissection of at least 1 supraaortic vessel or aortic arch true lumen collapse with $(\mathrm{M} 2+)$ or without (M2-) clinical symptoms of cerebral (stroke) or upper extremity (pulse deficit, pain, pallor, paraesthesia) malperfusion); and M3 (dissection or false lumen origin of at least 1 visceral, renal or 1 iliac artery or aortic true lumen collapse entailing functional closure of at least 1 visceral, renal or iliac artery offspring, with (M3+) or without (M3-) clinical symptoms of bowel (abdominal pain, ileus, bloody diarrhoea), kidney (anuria, renal failure signs) or lower extremity (pulse deficit, pain, pallor, paraesthesia) ischaemia.)

Application of the new classification: Take a dissection case in type A E1 M2+ M3-, as an example. Aortic dissection involved the ascending aorta, so the case could be classified in type A. The case could be described as type A E1 because the primary entry was located in the ascending aorta. The brachiocephalic trunk was dissected, and the patient suffered from stroke, so the case could be represented as type A E1 M2+. And, the inferior mesenteric arteries were involved without clinical symptoms, therefore the case could be characterized with type A E1 M2+ M3-.

Treatments: Ascending replacement, hemiarch replacement, total arch replacement, and hybrid procedures were performed in type A. The total arch replacement procedure includes arch replacement, elephant trunk, frozen elephant trunk, and stented elephant trunk. Hybrid procedures performed in type A included ascending replacement and thoracic endovascular repair (TEVAR) zone 0 . Some of the cases in type $B$ received conservative treatment, while others were treated with TEVAR, ascending replacement, hemiarch replacement, total arch replacement, and hybrid procedures. TEVAR performed in the two types included TEVAR zone 1, 2 and 3. TEVAR zone 0,1 , and 2 performed in all cases included fenestrated TEVAR and TEVAR with chimneys.

Statistical analyses: All data were analyzed by SPSS 24.0 software. The measurement data was reported as mean \pm standard deviation. To compare continuous variables, the Student's t-test was applied when equal distribution was present as tested by the Kolmogorov-Smirnov test. For unequally distributed variables, the Mann-Whitney rank sum test was used. Categorical variables were compared using the $\chi^{2}$ test. In the case of small group sizes $(N<5)$, the Fisher's exact test was used. The difference was statistically significant $(P<0.05)$.

\section{RESULTS}

Analysis of the type, entry and malperfusion classification system: Among 151 patients, $53.0 \%, 27.8 \%$, and $19.2 \%$ were type A, B, and non-A non-B, respectively. Among 80 cases with type A, there were $5 \%, 62.5 \%, 15 \%$, and $17.5 \%$ of cases with E0, E1, E2, and E3, respectively. Among 42 cases with type $\mathrm{B}$, there were $11.9 \%$ and $88.1 \%$ of cases with E0 and E3. And among 29 cases with type non-A non-B, there were $10.3 \%, 58.6 \%$, and $31.0 \%$ of cases with $\mathrm{E} 0, \mathrm{E} 2$, and E3, respectively. (Table 1) (Figure 1) M0 was found in $30.0 \%$, $59.5 \%$, and $13.8 \%$ of cases in type A, B, and non-A non-B, respectively. $\mathrm{M} 1$, with coronary artery malperfusion, only occurred in cases with type A, which included $5.0 \%$ and $1.3 \%$ of cases with M1+ and M1-. M2 occurred in type A and type non-A non-B. Type A M2 occurred in 44 cases, including $23.8 \%$ with $\mathrm{M} 2+$ and $31.3 \%$ with $\mathrm{M} 2-$. Type non-A non-B M2 also occurred in 22 cases, including $27.6 \%$ with $\mathrm{M} 2+$ and $51.7 \%$ with M2-. However, M3 occurred in all three types. There were 46 cases with type A M3, including $22.5 \%$ with $\mathrm{M} 3+$ and $55.0 \%$ with M3-. Type B M3 occurred in 17 cases, including $19.0 \%$ with $\mathrm{M} 3$ + and $21.4 \%$ with $\mathrm{M} 3$-. There were 8 cases with type non-A non-B M3, including $13.8 \%$ with $\mathrm{M} 3+$ and $13.8 \%$ with M3-, respectively.

Aortic surgery and outcomes: No patients with type A and type non-A non-B dissection were treated conservatively, while seven cases with type $B$ received conservative treatment. There were $6.3 \%$ of patients with type A who died of pericardial tamponade or hemorrhage, due to aortic dissection rupture before operation. The remaining 75 patients (93.8\%) with type A received surgical treatment. The ascending aorta was replaced in $93.8 \%$ of patients with type $\mathrm{A}$, in $2.4 \%$ with type $\mathrm{B}$, and in $6.9 \%$ with non-A non-B dissection $(P<0.001)$. The hemi-aortic arch was replaced in $27.5 \%$ of patients with type $\mathrm{A}$, in $4.8 \%$ with type $\mathrm{B}$, and in $6.9 \%$ with non-A non-B dissection $(P<0.001)$. The total aortic arch was replaced in $53.8 \%$ of patients with type A, in $4.8 \%$ with type $\mathrm{B}$, and in $13.8 \%$ with non-A non-B dissection $(P<0.001)$. In particular, dissection reversed to ascending aorta took place in two cases with type non-A non-B after fenestrated TEVAR, so ascending and total aortic arch replacement immediately were performed. Four cases in type A $(5.0 \%)$ received hybrid procedures, including ascending replacement 
Table 1. TEM classification of all 151 cases

\begin{tabular}{lccc}
\hline & Type $A(N=80)$ & Type $B(N=42)$ & Type non-A non-B (N=29) \\
\hline E (Entry) & & & $3(10.3 \%)$ \\
E0 & $4(5.0 \%)$ & $5(11.9 \%)$ & 0 \\
E1 & $50(62.5 \%)$ & 0 & $17(58.6 \%)$ \\
E2 & $12(15.0 \%)$ & 0 & $9(31.0 \%)$ \\
E3 & $14(17.5 \%)$ & $37(88.1 \%)$ & $4(13.8 \%)$ \\
M (Malperfusion) & & & 0 \\
M0 & $24(30.0 \%)$ & $25(59.5 \%)$ & 0 \\
M1+ & $4(5.0 \%)$ & 0 & $8(27.6 \%)$ \\
M1- & $1(1.3 \%)$ & 0 & $15(51.7 \%)$ \\
M2+ & $19(23.8 \%)$ & 0 & $4(13.8 \%)$ \\
M2- & $25(31.3 \%)$ & 0 & $4(13.8 \%)$ \\
M3+ & $18(22.5 \%)$ & $8(19.0 \%)$ & $9(21.4 \%)$ \\
M3- & $28(35.0 \%)$ & \\
\hline
\end{tabular}

+ with clinical symptoms; - without clinical symptoms. Categorical variables are given as counts and percentages.

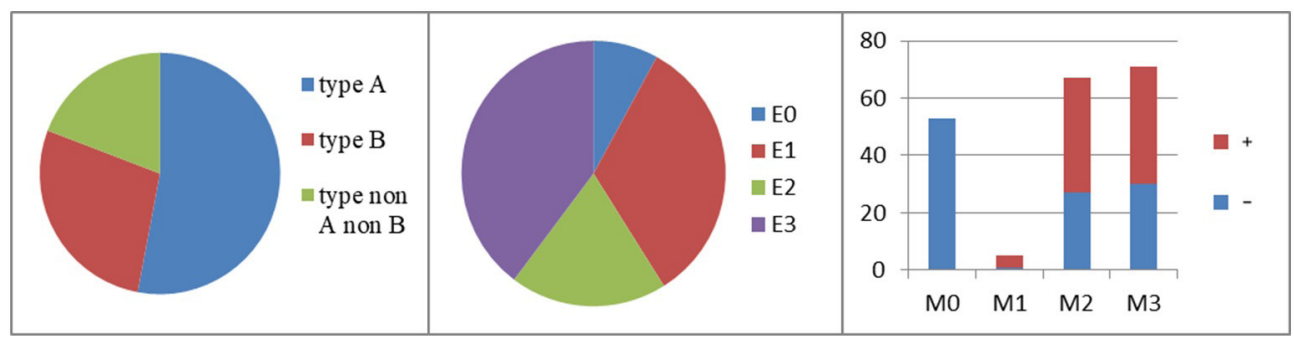

Figure 1. TEM classification for 151 patients

and fenestrated TEVAR zone 0 . Most of TEVAR zone 1 and 2 procedures were performed with type non-A non-B with $31.0 \%$ and $55.2 \%$, respectively. Only $9.5 \%$ of patients with type $\mathrm{B}$ received TEVAR zone $2(P<0.001)$. TEVAR zone 3 procedures were performed in $12.0 \%$ of patients with type A (in addition to ascending replacement), in $73.8 \%$ with type $\mathrm{B}$ and in $13.8 \%$ with non-A non-B $(P<0.001)$.

The in-hospital mortality rate was $8.8 \%, 2.4 \%$, and $3.4 \%$ in patients with type $\mathrm{A}, \mathrm{B}$, and non-A non- $\mathrm{B}$, respectively $(P<0.05)$. Nine patients died within six months after surgery because of multiple organ failure and severe pulmonary infection. Among 34 cases, there were occurrences of various postoperative neurological complications among the groups $-33.8 \%, 7.1 \%$, and $13.8 \%$ with type A, B and non-A non-B, respectively $(P<0.05)$. Among 16 cases with postoperative abdominal complications, such as abdominal pain and diarrhea, these took place in $12.5 \%, 7.1 \%$, and $10.3 \%$ of patients with type A, B, and non-A non-B, respectively ( $P$ $>0.05$ ). Postoperative continuous renal replacement therapy (CRRT) because of renal dysfunction was performed in 19 cases, including $21.3 \%, 2.4 \%$, and $3.4 \%$ of patients with type $\mathrm{A}, \mathrm{B}$, and non-A non-B, respectively $(P>0.05)$. (Table 2$)$
Entry location and outcomes: The entry was located in the ascending aorta in most of our patients with type A dissection (type A E1) (62.5\%), while $15.0 \%$ with type A E2, $17.5 \%$ with type A E3, and $5.0 \%$ with type A E0. In cases with type B, there was $11.9 \%$ with type B E0 and $88.1 \%$ with type B E3. In cases with type non-A non-B, there was $10.3 \%$ with type non-A non-B E0, 58.6\% with type non-A non-B E2, and 31.0\% with type non-A non-B E3 (Table 1). Among all patients, there were $6.0 \%, 3.4 \%$, and $1.7 \%$ with entry in ascending aorta (E1), in aortic arch (E2), and in descending aorta (E3), respectively $(P<0.05)$. Of patients with type E0, $33.3 \%$ received conservative treatment $(P<$ $0.001)$. The ascending aorta was replaced in $16.7 \%$ of cases with type E0, $94.0 \%$ with type E1, $41.4 \%$ of patients with type E2, and $11.7 \%$ patients with type E3 $(P<0.001)$. The hemi-aortic arch was replaced in $8.3 \%$ of patients with type E0, $44.0 \%$ with type E1, and $10.3 \%$ of patients with type E2 $(P<0.01)$. The total aortic arch was replaced in $8.3 \%$ of patients with type E0, $22.0 \%$ with type E1, $75.9 \%$ of patients with type E2, and $26.7 \%$ patients with type E3 $(P<0.01)$. TEVAR zone 0 procedures were performed in four cases with type A E3. TEVAR zone 1 procedures were 
performed in $8.3 \%$ of patients with type E0, $6.9 \%$ with type E2, and $10.0 \%$ with type E3. TEVAR zone 2 procedures were performed in $16.7 \%$ of patients with type E0, $6.9 \%$ with type $\mathrm{E} 2$, and $26.7 \%$ with type $\mathrm{E} 3(P<0.01)$. No case with type E1 received TEVAR zone 1 and TEVAR zone 2. TEVAR zone 3 procedures were performed in $25.0 \%$ of patients with type E0, $18.0 \%$ with type E1, and $53.3 \%$ with type E3 $(P<0.01)$, while these procedures were not chosen in cases with type E2.

The in-hospital mortality rate was $12.0 \%, 10.4 \%$, and $8.5 \%$ in patients with type E1, E2, and E3, respectively. Among 34 cases where postoperative neurological complications occurred, there were $8.3 \%, 20.0 \%, 41.4 \%$, and $16.7 \%$ of patients with type E0, E1, E2, and E3, respectively $(\mathrm{P}<0.05)$. Among 16 cases with postoperative abdominal complications, there were $14.0 \%, 13.8 \%$, and $8.3 \%$ of patients with type E1, E2, and E3 $(P>0.05)$. Postoperative CRRT was performed in 19 cases, including $26 \%, 6.9 \%$, and $8.3 \%$ of patients with type E1, E2, and E3, respectively $(P<0.01)$. (Table 3$)$

Malperfusion and outcomes: The preoperative mortality rate was $40.0 \%, 4.5 \%$, and $5.6 \%$ of the patients with type M1, M2, and M3, respectively $(P<0.01)$. There were $4.8 \%$ and $4.2 \%$ of patients with type M0 and type M3 who received conservative treatment. Cases with type E0 were found in $10.8 \%$ and $4.2 \%$ of patients with type M0 and type M3. Cases with type E1 that had entry in the ascending aorta were found in $38.6 \%$, $40.0 \%, 46.3 \%$, and $26.8 \%$ of patients with type M0, M1, M2, and $\mathrm{M} 3$, respectively $(P>0.05)$. Cases with type $\mathrm{E} 2$ were found in $21.7 \%, 20.0 \%, 38.8 \%$, and $16.9 \%$ of patients with type $\mathrm{M} 0$, $\mathrm{M} 1, \mathrm{M} 2$, and $\mathrm{M} 3$, respectively $(P<0.05)$. And cases with type E3 were found in $28.9 \%, 10.4 \%$, and $50.7 \%$ of patients with type M0, M2, and M3 $(P<0.01)$. Procedures of coronary revascularization, such as coronary artery bypass grafting, Bentall procedure and Wheat's procedure, were performed in $9.6 \%, 60.0 \%$, $19.4 \%$, and $15.5 \%$ of patients with type M0, M1, M2, and M3, respectively $(P<0.01)$. Total arch replacement was performed in $26.5 \%, 60.0 \%, 56.7 \%$, and $18.3 \%$ of patients with type $\mathrm{M} 0$, M1, M2, and M3 $(P<0.01)$. Procedures of revascularization of visceral, renal, or iliac artery by bypass grafts or stents were performed in nine cases with type M3.

The in-hospital mortality rate was $20.0 \%, 10.4 \%$, and $8.5 \%$ in patients with type $M 1, M 2$, and $M 3$, respectively $(P<0.05)$. Among 34 cases with postoperative neurological complications, there were $7.2 \%, 20.0 \%, 28.4 \%$, and $11.3 \%$ of patients with type M0, M1, M2, and M3, respectively ( $P$ $<0.05$ ). Among 16 cases with postoperative abdominal complications, there were $2.4 \%, 20.0 \%, 11.9 \%$, and $15.5 \%$ of patients with type M0, M1, M2, and M3 $(P<0.05)$. Postoperative CRR was performed in 19 cases, including $2.4 \%$, $20.0 \%, 7.5 \%$, and $25.4 \%$ of patients with type M0, M1, M2, and $\mathrm{M} 3$, respectively $(P<0.05)$.

\section{DISCUSSION}

With the continuous progress of medical examination and treatment technology, aortic operations have attained enormous progress in diagnosis and treatment in the past 50 years.
We are now able to replace every aortic segment in an open or an endovascular procedure. Aortic operations have benefited from new procedures, e.g., prosthesis replacement, classic and frozen elephant trunk techniques, endovascular stents, and hybrid procedures. Both the commonly used Stanford classification system and Debakey classification system could not provide more essential information on different types of aortic dissections. The location of the primary entry of aortic dissection and the end-organ malperfusion had aroused concern increasingly [Colli 2018; Shi 2016; Khoynezhad 2010; Weiss 2012; Geirsson 2007; Kreibich 2019; Di Eusanio 2013]. A novel classification system, TEM classification, was proposed by Sievers et al. [Sievers 2020]. The parameters of this classification include the extension of the aortic dissection (A, B, and non-A non-B), entry location (E), and organ malperfusion (M). Different entry location and different organ malperfusion in cases even with the same Stanford type will cause significant differences in clinical symptoms and hemodynamics, which will lead to different medical treatment and surgical processes, and even result in different outcomes and prognosis.

Analysis of the type, entry and malperfusion classification system: We analyzed 151 patients with aortic dissection in our center between January 2019 and May 2020 and revealed that more than $50.0 \%$ of them suffered aortic type $\mathrm{A}$ and more than $60.0 \%$ of type A had primary entry in the ascending aorta. E2, entry located in the arch, occurred in cases with type $\mathrm{A}$ and non-A non-B, while $\mathrm{E} 3$ occurred in all three types, A, B, and non-A non-B. M2 occurred in cases with type $A$ and non-A non-B, while $\mathrm{M} 3$ occurred in all three types.

Aortic surgery: All patients with type A dissection in our group received ascending replacement. And more than $50 \%$ of these patients received total arch replacement, especially the patients with entry located in the arch (type E2) or descending (type E3). Ascending replacement with or without hemi-arch replacement could be performed in cases with type A E1, especially in the patients with high risk of deep hypothermic bypass circulation. Not all of the cases with type M0 should receive total arch replacement $(<30 \%)$, whereas more than half of the cases with type M2 should accept this procedure. Hybrid procedures, including ascending replacement and fenestrated TEVAR zone 0 procedure, were performed in four cases with type A E3, as the entry is located in the descending aorta. There is still some dispute about whether hybrid procedure should be chosen when the entry is located in ascending (type A E1) or in the arch (type A E2) [Spiliotopoulos 2018; Settepani 2015]. Most cases with type B were suggested to receive TEVAR [O'Donnell 2011]. Additionally, revascularization of visceral, renal, or iliac arteries should be performed concurrently in cases with type B M3. There are many choices of treatment for type non-A non-B, including TEVAR zone 1, TEVAR zone 2, TEVAR zone 3 , hemi or total arch replacement, and hybrid procedure [White 2011; Wang 2019]. Treatment for dissection in the arch is the key point. More than $50 \%$ of cases with type non-A non-B in our center received TEVAR zone 2 procedure, and most of them had entry in the descending (type non-A non-B E3). Two cases with type non-A non-B E2 received emergent total arch replacement because dissection tears reverse to ascending 
Table 2. Aortic surgery and outcomes

\begin{tabular}{lccc}
\hline & Type A $(N=80)$ & Type B $(N=42)$ & Type non-A non-B $(N=29)$ \\
\hline Preoperative death $(N=5)$ & 5 & 0 & 0 \\
Conservative treatment & 0 & 7 & 0 \\
Ascending replacement & $75(93.8 \%)$ & $1(2.4 \%)$ & $2(6.9 \%)$ \\
Hemi-arch replacement & $22(27.5 \%)$ & $2(4.8 \%)$ & $2(6.9 \%)$ \\
Total arch replacement & $43(53.8 \%)$ & $2(4.8 \%)$ & $4(13.8 \%)$ \\
TEVAR zone 0 & $4(5.0 \%)$ & 0 & 0 \\
TEVAR zone 1 & 0 & 0 & $9(31.0 \%)$ \\
TEVAR zone 2 & 0 & $31(73.8 \%)$ & $16(55.2 \%)$ \\
TEVAR zone 3 & $9(12.0 \%)$ & $1(2.4 \%)$ & $4(13.8 \%)$ \\
In-hospital deaths $(N=9)$ & $7(8.8 \%)$ & $3(7.1 \%)$ & $1(3.4 \%)$ \\
Neurological complications $(N=34)$ & $27(33.8 \%)$ & $3(7.1 \%)$ & $4(13.8 \%)$ \\
Abdominal complications $(N=16)$ & $10(12.5 \%)$ & $1(2.4 \%)$ & $3(10.3 \%)$ \\
Postoperative CRRT $(N=19)$ & $17(21.3 \%)$ & $1(3.4 \%)$ \\
\hline
\end{tabular}

Categorical variables are given as counts and percentages. Total arch replacement includes total arch replacement, elephant trunk, frozen elephant trunk and stented elephant trunk procedure.

TEVAR, thoracic endovascular aortic repair.

Tabl3 3. Entry location and outcomes

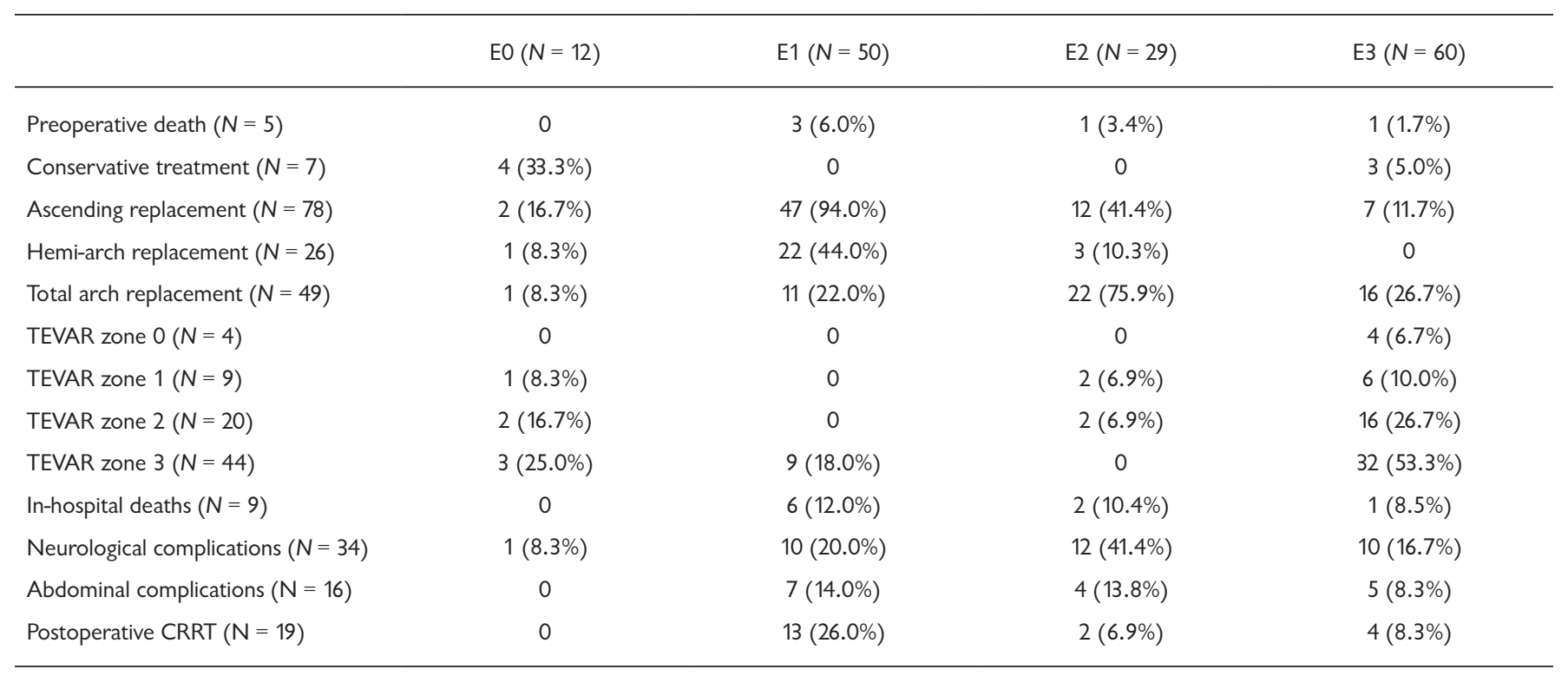

Categorical variables are given as counts and percentages 
Table 4. Malperfusion and outcomes

\begin{tabular}{|c|c|c|c|c|}
\hline Preoperative death $(N=5)$ & 0 & $2(40.0 \%)$ & $3(4.5 \%)$ & $4(5.6 \%)$ \\
\hline EO $(N=12)$ & $9(10.8 \%)$ & 0 & 0 & $3(4.2 \%)$ \\
\hline $\mathrm{E} 1(N=50)$ & $32(38.6 \%)$ & $2(40.0 \%)$ & $31(46.3 \%)$ & $19(26.8 \%)$ \\
\hline E3 $(N=60)$ & $24(28.9 \%)$ & 0 & $7(10.4 \%)$ & $36(50.7 \%)$ \\
\hline Coronary revascularization $(N=19)$ & $8(9.6 \%)$ & $3(60.0 \%)$ & $13(19.4 \%)$ & $11(15.5 \%)$ \\
\hline Total arch replacement $(N=49)$ & $22(26.5 \%)$ & $3(60.0 \%)$ & $38(56.7 \%)$ & $13(18.3 \%)$ \\
\hline Revascularization of visceral, renal, or iliac artery $(N=9)$ & 0 & 0 & 0 & $9(12.7 \%)$ \\
\hline In-hospital deaths $(N=9)$ & 0 & $1(20.0 \%)$ & $7(10.4 \%)$ & $6(8.5 \%)$ \\
\hline
\end{tabular}

Categorical variables are given as counts and percentages. CRRT, continuous renal replacement therapy

during the TEVAR procedure. We suggest hemi or total arch replacement, and hybrid procedure should be considered in cases with type non-A non-B E2.

Entry: As the cases with type A E1 M1 tend to result in preoperative death, emergent surgery should be performed immediately. More than half of the patients with type non-A non-B also presented with an entry in the aortic arch and had dissection involvement of at least 1 supra-aortic vessel. As mentioned above, location of entry was very important for decision-making of hybrid procedure, especially in the cases with type A or non-A non-B. And we should pay more attention to the cases with $\mathrm{E} 1$ and $\mathrm{E} 2$, due to postoperative complications, like neurological complications, tend to occur in these cases.

Malperfusion: Malperfusion is a common and severe complication of aortic dissection and can involve many vital organs, resulting in cardiac ischemia, stroke, or lower extremity ischemia [Geirsson 2007; Kreibich 2019; Di Eusanio 2013]. It has been reported that $16 \%-34 \%$ of cases with dissections have malperfusion syndrome, and even the perioperative mortality rate up to $29 \% \sim 89 \%$ [Berretta 2018; Narayan 2017]. In our study, both the perioperative mortality rate and in-hospital mortality rate were high in cases with type M1. Neurological complications tended to occur in cases with type M2. And postoperative CRRT were performed in most cases with type M3. Some researchers recommend carrying out end-organ revascularization during operation, while others promote revascularization first [Vendell 2015; Van Bogerijen 2014]. In any case, we suggest relieving organ ischemia as soon as possible, especially the existence of severe ischemia of vital organs.

Limitations: The sample size needs to be expanded, especially the cases with type B and non-A non-B. Although the entry location was important to choose treatment methods, it could not be found by CTA scans in some cases before operation. The TEM classification needs to be reconfirmed during or after operation. The use of this classification is limited to some extent and needs to be improved by progress of imaging examination and technology.

\section{CONCLUSION}

The type, entry site, malperfusion classification, and TEM classification, provided the detail of important aspects of entry location and malperfusion and could be utilized as the supplement of the Stanford classification. It is hereby highly recommended to satisfy the development requirements of current technology, such as TEVAR and hybrid procedure, meanwhile, aid in decision-making and prognosis-judging.

\section{ACKNOWLEDGEMENT}

This work was supported by Department of Human Resource and Social Security of Shanxi province China (grant number 2019-29).

\section{REFERENCES}

Berretta P, Trimachi S, Patel HJ, et al. 2018. Malperfusion syndromes in type A aortic dissection: what we have learned from IRAD. J Vis Surg. 10:65-72.

Colli A, Carrozzini M, Francescato A, et al. 2018. Acute DeBakey Type I intimal tear in the arch: is total arch replacement the right choice? Interact Cardiovasc Thorac Surg. 26:84-90. 
Daily PO, Trueblood HW, Stinson EB, et al. 1970. Management of acute aortic dissections. Ann Thorac Surg. 10: 237-47.

Di Eusanio M, Trimarchi S, Patel HJ, et al. 2013. Clinical presentation, management, and short-term outcome of patients with type A acute dissection complicated by mesenteric malperfusion: Observations from the international registry of acute aortic dissection. J Thorac Cardiovasc Surg. 145:385-90.

Geirsson A, Szeto WY, Pochettino A, et al. 2007. Significance of malperfusion syndromes prior to contemporary surgical repair for acute type A dissection: outcomes and need for additional revascularizations. Eur J Cardiothorac Surg. 32:255-62.

Khoynezhad A, Walot I, Kruse MJ, et al. 2010. Distribution of intimomedial tears in patients with type B aortic dissection. J Vasc Surg. 52:562-8.

Kreibich M, Bavaria JE, Branchetti E, et al. 2019. Management of Patients With Coronary Artery Malperfusion Secondary to Type A Aortic Dissection. Ann Thorac Surg. 107:1174-80.

Narayan P, Rogers CA, Benedetto U, et al. 2017. Malperfusion rather than merely timing of operative repair determines early and late outcome in type A aortic dissection. J Thorac Cardiovasc Surg. 154:81-6.

O'Donnell S, Geotchues A, Beaver F, et al. 2011. Endovascular management of acute aortic dissections. J Vasc Surg. 54:1283-9.

Settepani F, Cappai A, Basciu A, et al. 2015. Hybrid Versus Conventional Treatment of Acute Type A Aortic Dissection. J Card Surg. 30:707-13.

Shi Y, Zhu M, Chang Y, et al. 2016. The risk of stanford type-A aortic dissection with different tear size and location: a numerical study. Biomed Eng Online. 15(Suppl 2):128.

Sievers HH, Rylski B, Czerny M, et al. 2020. Aortic dissection reconsidered: type, entry site, malperfusion classification adding clarity and enabling outcome prediction. Interact Cardiovasc Thorac Surg. 30:451-7.

Spiliotopoulos K, Preventza O, de la Cruz KI, et al. 2018. Management of expanding aortic arch aneurysm after hybrid endovascular and debranching repair. Eur J Cardiothorac Surg. 54:185-6.

Van Bogerijen GH, Williams DM, Patel HJ. 2014. TEVAR for complicated acute type B dissection with malperfusion. Ann Cardiothorac Surg. 3:423-7.

Vendell A, Frandon J, Rodiere M, et al. 2015. Aortic dissection with acute malperfusion syndrome: endovascular fenestration via the funnel technique. J Thorac Cardiovasc Surg. 150:108-15.

Wang W, Piao H, Wang Y, et al. 2019. Long-Term Outcomes of Hybrid Technique of Complicated Type BAorticDissection. Ann Thorac Surg. 107:1319-25.

Weiss G, Wolner I, Folkmann S, et al. 2012. The location of the primary entry tear in acute type B aortic dissection affects early outcome. Eur J Cardiothorac Surg. 42:571-6.

White RA, Miller DC, Criado FJ, et al. 2011. Report on the results of thoracic endovascula aortic repair for acute, complicated, type B aortic dissection at 30 days and 1 year from a multidisciplinary subcommittee of the Society for Vascular Surgery Outcomes Committee. Vasc Surg. 53:1082-90. 